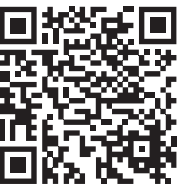
Simulador, adiestramiento médico, auscultación cardiaca.

Keywords: Simulator, medical training, cardiac auscultation.

* Departamento de Ingeniería en Sistemas Biomédicos, División de Ingeniería Mecánica e Industrial (DIMEI), Facultad de Ingeniería de la UNAM. Ciudad de México, México.

Recibido: 17/02/2020

Aceptado: 30/11/2020

doi: $10.35366 / 97904$

\section{Diseño de un simulador de paciente para auscultación cardiaca}

\author{
Design of a patient simulator for cardiac auscultation
}

\author{
Luis Jiménez-Ángeles, * Andrés Rosario-Rojas,* Sergio Alejandro Viaña-Fragoso*
}

\section{RESUMEN}

El uso de simuladores en el adiestramiento médico permite reproducir diferentes condiciones fisiológicas en un ambiente controlado, reproducible y cuantificable; esto favorece el proceso de enseñanza-aprendizaje sin riesgo a los usuarios. En este documento se describe el diseño y desarrollo de un simulador de paciente que permite reproducir el proceso de auscultación cardiaca. El simulador propuesto está constituido por un modelo anatómico del cuerpo humano con medidas antropométricas similares a las de un hombre adulto y un circuito electrónico que controla el sonido cardiaco asociado al sitio donde se realiza la auscultación. El modelo anatómico se construyó mediante el modelado de la caja torácica y el cráneo. La piel sintética fue construida con silicón y ajustada a la caja torácica con espuma flexible expandible para simular los músculos y el tejido adiposo. El circuito electrónico controla los módulos de reproducción de audio y permiten el almacenamiento de diferentes y variadas condiciones fisiológicas. Así también, el circuito electrónico controla cinco sensores táctiles de presión, los cuales activan indicadores luminosos cuando la colocación del estetoscopio en el foco de auscultación es la correcta. Una ventaja competitiva de nuestro desarrollo respecto a los comercialmente disponibles es la apertura que tiene para realizar modificaciones en su operación, estructura y conformación; además del bajo costo asociado en su fabricación. Estas características permitirán expandir la funcionalidad para registrar y monitorear diversas variables fisiológicas, tales como las relacionadas con la actividad eléctrica muscular, cerebral, respiratoria, pulmonar o mecánica. La futura validación del simulador propuesto permitirá evaluar su capacidad de incrementar las habilidades de los médicos en formación.

\section{ABSTRACT}

The use of simulators in medical training allows the reproduction of different physiological conditions in a controlled, reproducible and quantifiable environment; this favors the teaching-learning process without risk to the users. This document describes the design and development of a patient simulator that makes it possible to reproduce the process of cardiac auscultation. The proposed simulator consists of an anatomical model of the human body with man and an electronic circuit that controls the heart sound associated with the site where the auscultation is performed. The anatomical model was built by modeling the ribcage and skull. The synthetic skin was built with silicone and adjusted to the ribcage with flexible expandable foam to simulate the muscles and the adipose tissue. The electronic circuit controls the audio reproduction modules and allows the storage of different and varied physiological conditions. Also, the electronic circuit controls five tactile pressure sensors which activate light indicators when the placement of the stethoscope in the auscultation focus is correct. A competitive advantage of our development with respect to the commercially available ones is the flexibility to make modifications in its operation, structure and conformation; besides the low cost associated to its manufacture. These features will allow expanding the functionality to record and monitor various physiological variables such as those related to electrical muscle activity, brain, respiratory, lung or mechanical. The future validation of the proposed simulator will allow to evaluate its capacity to increase the skills of doctors in training. anthropometric measurements similar to those of an adult

\section{INTRODUCCCIÓN}

E proceso de auscultación cardiaca es una de las habilidades clínicas más importantes de exploración física, ya que permite identificar información útil para el diagnóstico de diferentes patologías tales como valvulopatías, enfermedades congénitas, falla cardiaca, entre otras. ${ }^{1-3}$ Sin embargo, existen diversos estudios que indican que las habilidades de auscultación cardiaca son insuficientes no sólo
Citar como: Jiménez-Ángeles L, Rosario-Rojas A, Viaña-Fragoso SA. Diseño de un simulador de paciente para auscultación cardiaca. Simulación Clínica. 2020; 2 (3): 147-154. https://dx.doi.org/10.35366/97904 
en estudiantes de medicina, sino también en especialistas de medicina interna y medicina familiar. ${ }^{4-6}$ Por lo que es crucial para los médicos contar con simuladores de paciente para recrear el proceso de auscultación cardiaca con el objetivo de adquirir o reforzar las habilidades y competencias en la identificación de los sonidos cardiacos.

Los simuladores de paciente son utilizados como herramientas educativas que les permiten a los estudiantes de medicina desarrollar habilidades profesionales y reconocer sus áreas de oportunidad. El Doctor David Gaba definió a la simulación como un proceso instruccional que reemplaza el contacto con pacientes reales mediante el uso de modelos artificiales o virtuales, con el objetivo de recrear diferentes escenarios clínicos en un ambiente realista con propósitos de evaluación y retroalimentación. ${ }^{7}$ Una simulación apropiada recrea un ambiente educativo ideal en el que las actividades de aprendizaje desarrolladas son consistentes, estandarizadas, predecibles, reproducibles y seguras. Dicho ambiente educativo ideal potencializa el aprendizaje a través de la experimentación con la posibilidad de regresar a una práctica específica en cualquier momento. ${ }^{8}$

Los simuladores de pacientes de alta fidelidad son aquéllos con los que, además de la práctica de procedimientos, también permiten a los educadores configurar diferentes escenarios en el que los alumnos pueden cometer errores que generen situaciones críticas o fatales, pero sin riesgo hacia el paciente. En dichos escenarios se pone mayor atención en la interacción y el comportamiento humano ante circunstancias adversas. ${ }^{9}$

La incorporación de nuevas tecnologías a los simuladores de alta fidelidad es uno de los campos científicos más prometedores e innovadores al permitir la completa conjugación entre las áreas de ingeniería y medicina. ${ }^{10} \mathrm{El}$ concepto de incorporar simuladores de pacientes como una herramienta educativa no es una idea nueva; sin embargo, su uso se ha intensificado y diversificado en organizaciones prestadoras de servicios de salud, se han hecho asequibles para personal médico de diferentes niveles y especialidades, se han incorporado en los programas de capacitación y adiestramiento en compañías aseguradoras y se ha incrementado su uso en compañías que suministran productos de atención a la salud. ${ }^{11}$

Dado un incremento en el uso y la demanda de los simuladores utilizados en el adiestramiento médico, es evidente la necesidad de incrementar y fomentar el desarrollo de proyectos tecnológicos, así como de recursos humanos que hagan mejoras significativas en su diseño, funcionalidad, grado de especialización y costos. Este documento describe el diseño y desarrollo de un simulador de paciente para el adiestramiento en el proceso de auscultación cardiaca mediante la generación de ruidos cardiacos normales y patológicos. Con la incorporación de esta propuesta tecnológica como herramienta complementaria en la formación y adiestramiento médico se pretende ofrecer sistemáticamente a todos los estudiantes, bajo condiciones controladas, la misma experiencia simulada: auscultar el mismo ruido cardiaco, disponible en una librería de opciones, que independiza al estudiante de la oportunidad de interactuar con un paciente con una patología determinada, estandarizando la experiencia para todos los estudiantes.

\section{MATERIAL Y MÉTODOS}

El simulador se desarrolló considerando dos bloques principales:

a) El diseño de un modelo anatómico que le proporcione al usuario la experiencia de interactuar de manera aproximada a la situación real con un humano.

b) El desarrollo de un circuito electrónico que reproduzca los sonidos cardiacos como respuesta al correcto posicionamiento del estetoscopio sobre el punto de auscultación y un sistema de retroalimentación al usuario.

\section{Modelo anatómico}

El modelo anatómico permite realizar las maniobras de auscultación en cinco puntos o focos de auscultación (aórtico accesorio, aórtico, mitral, pulmonar y tricúspide), los cuales están anatómicamente distribuidos considerando las proporciones anatómicas de un sujeto adulto estándar. ${ }^{12}$ La ubicación física de los focos de auscultación se realiza mediante la palpación e identificación de determinados espacios intercostales del tórax, en los cuales al momento de presionarlos con el estetoscopio se accionan interruptores mecánicos sensibles a presión, que originarán la activación de indicadores visuales como señal de la correcta localización anatómica de foco de auscultación.

Para el diseño del modelo anatómico se consideraron los siguientes puntos:

1) La piel sintética debe cubrir en una sola pieza la caja torácica y el cráneo. 
2) Los brazos y piernas deberán ser independientes al torso, articulados y con posibilidad de unirse al cuerpo si así se requiere en un futuro.

3) El interior del modelo anatómico tendrá un contenedor para el circuito electrónico de control del simulador.

\section{Modelado}

Mediante manufactura aditiva tridimensional, utilizando imágenes de resonancia magnética del torso de un voluntario hombre adulto estándar, se reprodujeron anatómicamente los huesos que componen la caja torácica y el cráneo. Se fijó el modelo de la caja torácica y el cráneo en una base para evitar caídas o movimientos indeseados y posteriormente se cubrió con plastilina epóxica para modelarlos a detalle. En la Figura 1 se puede observar un diagrama del proceso de modelado.

Molde y vaciado

Una vez realizado el modelado a detalle, se desarrolló un molde con material de silicón colocando registros en distintos puntos del torso y cubriendo el exterior con vendas de yeso, dando origen a
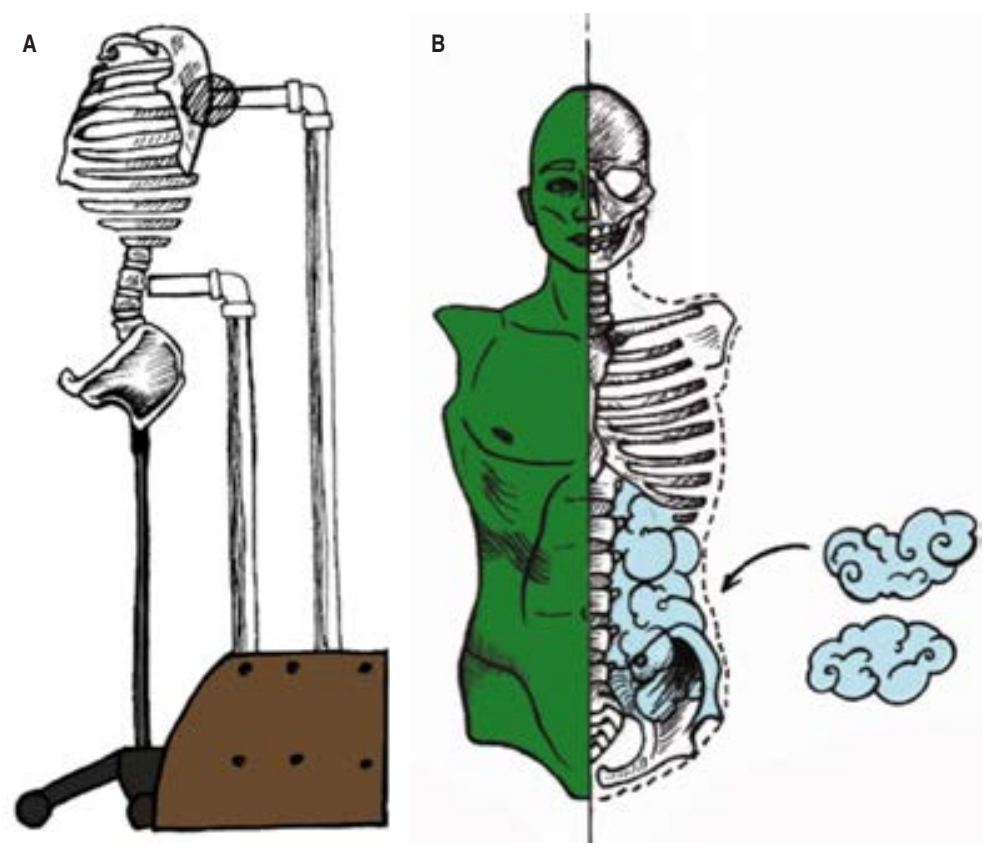

Figura 1: A) Ajuste y sujeción de la caja torácica a una base para evitar caídas o movimientos durante el proceso de modelado. B) Cubrimiento de la caja torácica y el cráneo con plastilina epóxica para detallar las características y proporciones anatómicas a detalle. una carcasa que evitará el movimiento del molde y su posible deformación. El molde se reforzó con bastones de madera. En la Figura $2 \mathrm{~A}$ se puede observar un diagrama del diseño del molde.

Una vez desmontado el molde, se removió la plastilina y el relleno utilizados con el fin de adaptarlo para que quede dentro del prototipo final. En la Figura $2 B$ se esquematiza una vista frontal de molde y su interior.

Utilizando el mismo silicón que se usó para elaborar el molde y pigmentos especiales, se modeló la piel del simulador en el interior del molde, agregando capas uniformes hasta obtener el grosor deseado. En esta última etapa se consideró el diseño de un espacio en el interior del simulador para colocar los sistemas electrónicos.

La piel sintética se pintó utilizando una mezcla a base de silicón compatible. La mezcla se aplicó en capas con diferentes tonalidades, haciendo énfasis en las zonas vasculares de la cara y el tórax. El orden de las tonalidades fue de claro a obscuro, aplicando tres tonalidades rojas seguidas de una café, posteriormente una tonalidad azul, una amarilla y finalmente se retocó para obtener un color homogéneo.

El pigmento fue aplicado con espuma y aerógrafo según la zona en donde se fuera a pintar. De igual manera, se utilizó silicón y pigmento para resanar y rellenar zonas que habían quedado al descubierto o que se habían roto durante el desmolde.

\section{Diseño del circuito electrónico de control}

El diseño del sistema electrónico se realizó considerando que deberá ser capaz de obtener una señal de la correcta posición del estetoscopio con el cual se simulará la exploración cardiaca en el modelo anatómico, lo que dará origen a la reproducción de los sonidos cardiacos. Si la localización del estetoscopio es la correcta, se informará visualmente al usuario con un LED indicador, lo que permitirá una retroalimentación rápida en términos de mejorar la habilidad en su entrenamiento.

En la Figura 3 se representa el diseño completo del circuito electrónico que controlará la operación del simulador.

En el diseño del circuito electrónico se consideró que las diferentes patologías a simular serán seleccionadas por medio de una aplicación, ejecutándose en una tableta con el sistema operativo Android. La aplicación se comunicará de forma inalámbrica mediante el protocolo Bluetooth con 

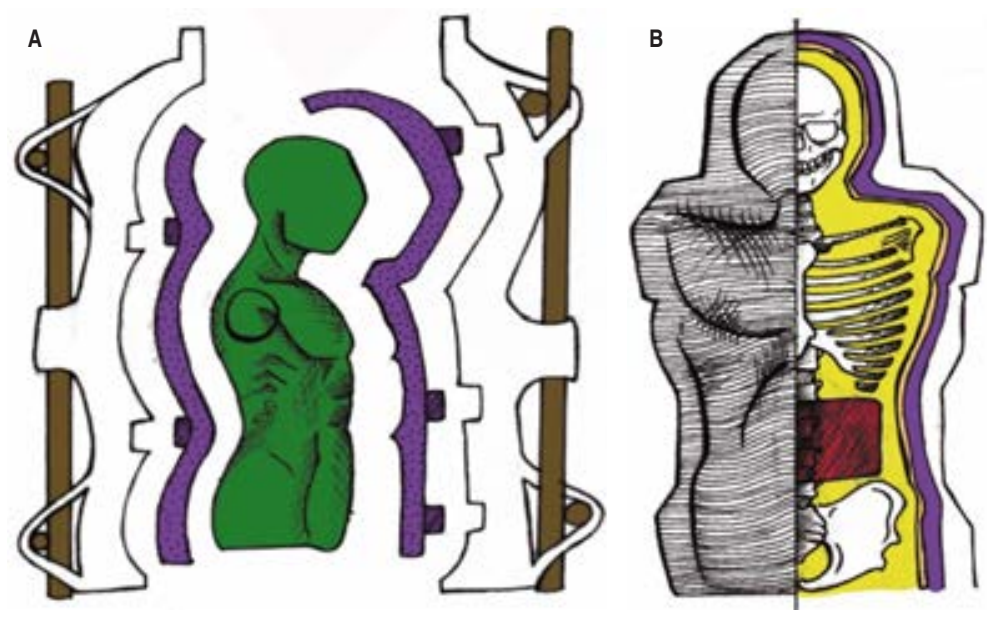

Figura 2: A) Se esquematiza el diseño del molde, donde la superficie en color verde representa el modelado a detalle del torso y la cabeza, el color morado representa el registro de silicón mientras que la zona blanca la carcasa de yeso. En café se pueden observar los soportes de madera. B) Se esquematiza una vista frontal del molde, con un corte del lado derecho donde se puede apreciar su interior. La superficie roja representa el contenedor de los componentes electrónicos, la amarilla el lugar que se cubrirá con espuma y el color durazno la piel sintética. un microcontrolador situado dentro del simulador de paciente. El microcontrolador configurará la operación de siete módulos de reproducción de audio MP3 (DFPlayer Mini) mediante comunicación serial asíncrona. Los módulos de reproducción seleccionarán una determinada pista de audio de las diferentes patologías almacenadas en su respectiva tarjeta de memoria microSD y la señal de audio será reproducida en bocinas de 8 ohms y de 15 milímetros de diámetro de propósito general. Por cada punto de auscultación será colocada una bocina.

En cada punto de auscultación se colocarán sensores táctiles de presión del tipo FSR 400, los cuales tienen un área activa de 5.08 milímetros y un rango lineal de sensibilidad de 0.2-20 Newtons. Cuando el usuario presione los sensores táctiles de presión mediante la colocación del estetoscopio, se enviará una retroalimentación visual mediante un LED, indicando que el sitio de auscultación fue el correcto.

Para el acondicionamiento de las señales de alimentación se consideró en el diseño una fuente de alimentación robusta en términos de que pueda ser operada con baterías o con el suministro de la toma de corriente.

El circuito electrónico fue modelado utilizando un programa de código abierto, para posteriormente generar la placa de circuito impreso.

\section{RESULTADOS}

\section{Modelo anatómico}

En la Figura 4 se ilustra el resumen de los resultados obtenidos en la construcción del modelo anatómico.

La Figura $4 \mathrm{~A}$ ilustra el resultado de la manufactura aditiva tridimensional del tórax y el cráneo de un sujeto voluntario adulto con medidas antropométricas estándar, utilizando imágenes de resonancia magnética. Ambos moldes fueron colocados en una base para evitar movimientos durante el proceso de modelado a detalle con plastilina epóxica (Figura 4B). En la Figura 4C se ilustra el resultado del molde con material de silicón (kit Dragon skin 20, Smooth-On Inc.), y en la Figura 4D se ilustra el reforzamiento del molde con vendas de yeso y bastones de madera. Una vez construido el molde, se realizó el proceso de fabricación de la piel sintética vaciando material de silicón de platino (kit Dragon skin FX-Pro, Smooth-On Inc.) en el molde y ajustando las dimensiones al cráneo y la caja torácica con espuma flexible expandible (kit Flex Foam-IT, Smooth-On Inc.), como se ilustra en las Figuras $4 E$ y F. La piel sintética se pintó con un aerógrafo y se utilizaron los pigmentos de color base silicón (Silc Pig, Smooth-On Inc.). Para la colocación del circuito electrónico controlador del simulador se consideró un espacio amplio en la espalda del maniquí con la tapa ajustable y sujeta a presión. La Figura 4G ilustra la piel sintética resultante y el espacio para el circuito electrónico. En la Figura $4 \mathrm{H}$ se ilustran las dimensiones finales del modelo anatómico.

El modelo anatómico del simulador permite la identificación mediante palpación de todos los espacios intercostales en el tórax. Dichos espacios intercostales tienen correlación anatómica con los del tórax de un adulto estándar y son fundamentales durante el entrenamiento médico para la localización de los focos específicos de auscultación cardiaca.

Los materiales utilizados en la construcción de la piel sintética son de alta calidad y se utilizan comúnmente en la industria cinematográfica para la creación de moldes y efectos especiales, lo que permite una mejor recreación de un escenario real de auscultación clínica.

El rellenar el modelo anatómico con espuma flexible expandible permitió simular los músculos y el tejido adiposo que los rodea, así como proporcionar mayor firmeza y rigidez al ensamble de la caja torácica con la piel sintética. 


\section{Circuito electrónico de control}

En la Figura $5 A$ se ilustra el diseño y una fotografía del circuito de control de los módulos de reproducción de audio (DFPlayer Mini, SKU:DFR0299Robot Wiki) que están conectados a las bocinas de 8 ohms y 15 milímetros de diámetro (CDMG 15008-03A, CUI Inc.). El control es ejecutado por medio de un microcontrolador ATmega2560 $\left(\right.$ Arduino $\left.{ }^{\circledR}\right)$, colocado en la parte posterior de la placa de circuito impreso. En la imagen se observa la disposición de los interruptores mecánicos que permiten seleccionar de forma manual el audio a ser reproducido; sin embargo, la selección y configuración de los audios están diseñados para que sea por medio de una aplicación que se ejecute sobre una tableta con sistema operativo Android en comunicación inalámbrica con el microcontrolador.

En la Figura $5 B$ se ilustra el diseño y una fotografía del circuito de control de los sensores táctiles de presión (FSR 402, Interlink electronics), los cuales son activados cuando el médico estudiante coloca y oprime la superficie del estetoscopio en un punto de auscultación específico. Esto origina la activación de una señal visual mediante un diodo emisor de luz (LED, Light Emitting Diode) específico. En la parte derecha de la Figura 5B también se ilustra la disposición de los transistores de potencia que ajustan y regulan el voltaje de entrada al circuito.

Los resultados preliminares de la evaluación de funcionamiento del circuito de control permiten identificar las siguientes ventajas:
- Se puede simular una sesión de auscultación cardiaca en la cual pueden intervenir hasta cinco estudiantes, ya que los sonidos cardiacos son reproducidos por cinco bocinas independientes.

- Se pueden almacenar diferentes y variadas patologías cardiacas, ya que cada módulo de reproducción de audio cuenta con su propia memoria de almacenamiento.

- Los sensores táctiles de presión permiten la auscultación con cualquier modelo de estetoscopio clínico.

- Se tiene una retroalimentación directa de la correcta colocación del estetoscopio en el foco de auscultación mediante el uso de indicadores luminosos, lo que facilita la adquisición de destrezas y habilidades de forma rápida e independiente de un supervisor.

El diseño del circuito electrónico de control permitió utilizar sistemas y componentes electrónicos de alta calidad y de bajo costo, lo que favorece la expansión de las capacidades del simulador en la evaluación de otras variables fisiológicas, tales como la respiración, la tensión arterial, el registro de potenciales bioeléctricos del sistema cardiaco, muscular o cerebral.

\section{DISCUSIÓN}

Los simuladores de paciente han sido utilizados con el objetivo de ayudar a los médicos en formación en la adquisición de aptitudes y habilidades en la exploración del corazón y los pulmones. En la literatura se han descrito diversos simuladores

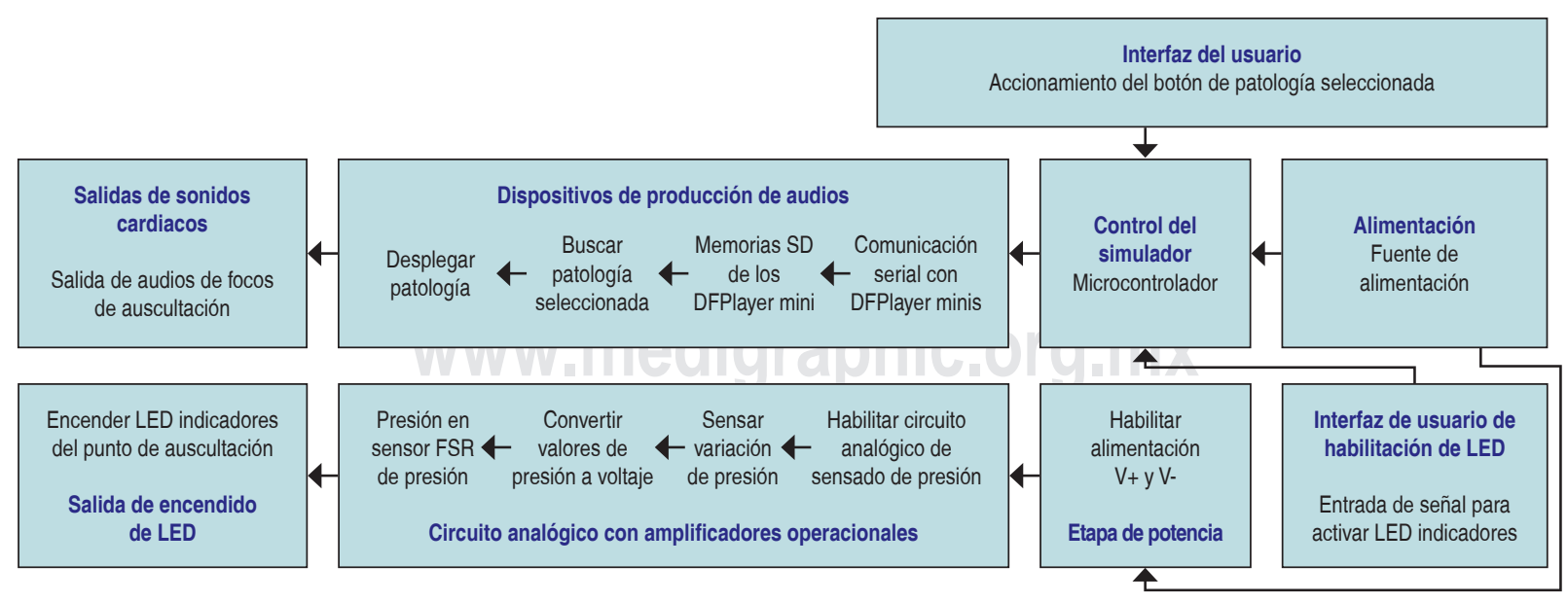

Figura 3: Diagrama a bloques del circuito electrónico que controla la operación del simulador. 



con la capacidad de simular diferentes condiciones o patologías, principalmente modificando parámetros que caracterizan los sonidos respiratorios, los sonidos cardiacos y la señal de la onda de pulso. ${ }^{13-16}$ Los reportes de la validación de la utilidad de los simuladores de paciente han mostrado que el usarlos mejora de forma significativa las habilidades y los conocimientos de los estudiantes en comparación con aquellos que sólo han practicado con pacientes, por lo que se recomienda incorporarlos a los programas de formación y capacitación del personal médico y paramédico. ${ }^{17,18}$

Existen diferentes simuladores de paciente disponibles en el mercado. ${ }^{19}$ Las principales características de los simuladores de torso consultados, similares al que proponemos en este proyecto (R10001 y R11000 de Erler-Zimmer, M164 de Sakamoto Model Corp, AN1142 de Adam Roully y 6950.11 de Altay Scientific) son que permiten la evaluación de los sonidos cardiacos y pulmonares en condiciones normales y patológicas. Los más costosos proponen evaluar sonidos intestinales y diferentes soplos. Así también, permiten programar la frecuencia cardiaca y respiratoria para recrear diferentes condiciones mediante un control remoto o una computadora. La mayoría brinda la posibilidad de enviar los diferentes sonidos a bocinas externas para la enseñanza en grupos.

En nuestra propuesta, al igual que en los disponibles en el mercado, los sitios de auscultación cardiaca no son visibles al estudiante, lo que favorece el adiestramiento en la palpación de las diferentes regiones anatómicas para la identificación de los diferentes focos. Nuestra propuesta no depende de un estetoscopio específico, mientras que en varias versiones comerciales se agrega un costo adicional por cada estetoscopio que es específico para la marca y modelo de simulador.

En las hojas de datos de los simuladores comerciales se tiene una cantidad limitada de patologías o condiciones a recrear, mientras que en nuestra propuesta la configuración y cantidad de sonidos sólo está limitada al espacio en las 
memorias de los controladores de audio; por lo tanto, prácticamente hay una cantidad ilimitada de condiciones y situaciones normales y patológicas que se pueden recrear.

Una de las principales ventajas de nuestra propuesta es que todos los elementos que componen el modelo anatómico están disponibles en el mercado y son relativamente accesibles en costo; por lo que, con las habilidades y experiencia obtenidas durante el desarrollo de este proyecto, se puede replicar el proceso para completar el maniquí con las extremidades superiores e inferiores, lo que posibilita el desarrollo de modelos anatómicos para fines distintos a los de la auscultación cardiaca e incluso para reproducir modelos de otras especies.

Resulta importante mencionar que una de las principales limitaciones de nuestra propuesta se focaliza en el proceso de manufactura del modelo anatómico, ya que la mayor parte del modelado y registro se realizó con técnicas artesanales; es un área de oportunidad a mejorar en caso de que se tenga la necesidad de producción en serie.

\section{CONCLUSIONES}

En este documento se describe el diseño y desarrollo de un simulador de paciente que permite reproducir el proceso de auscultación cardiaca mediante la identificación de los cinco
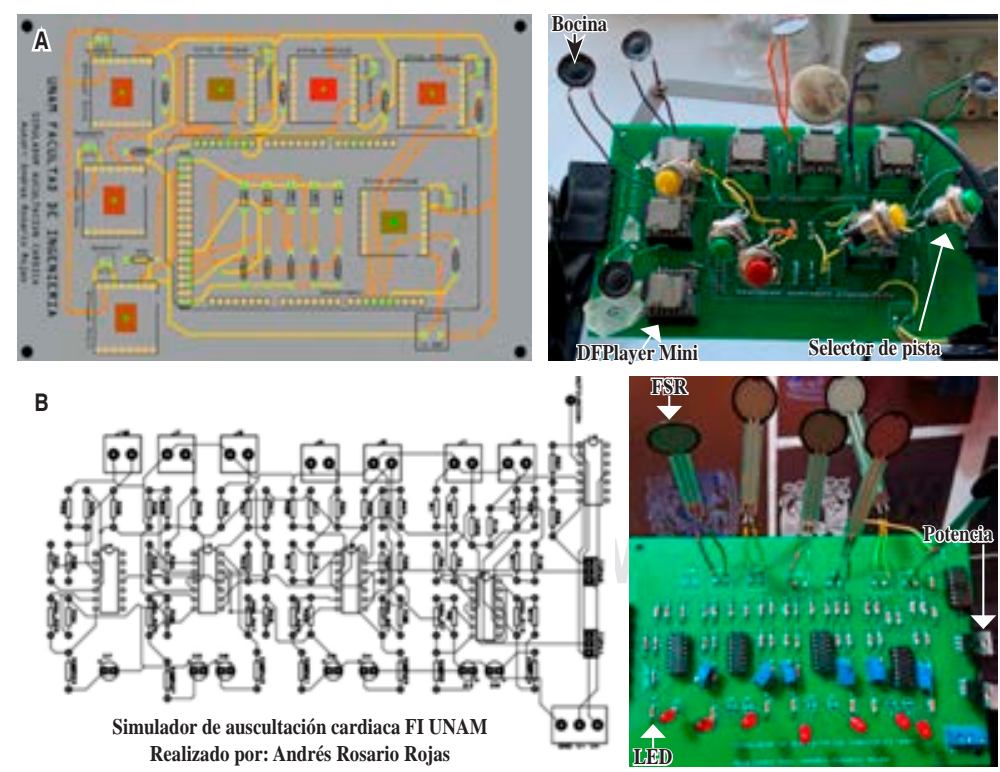

Figura 5: Diseño y fotografías del circuito de control de los módulos reproductores de audio (A), y de los sensores táctiles de presión, asi como del circuito de potencia (B). principales focos situados en la superficie externa del tórax de un modelo anatómico con medidas antropométricas de un hombre adulto con características anatómicas estándar. La piel sintética del modelo anatómico permite una mejor aproximación de un escenario real de auscultación clínica. La impresión de la caja torácica por medio de manufactura aditiva tridimensional permite la identificación precisa de los diferentes espacios intercostales necesarios para la ubicación de los focos de auscultación, además el espacio entre la piel sintética y la caja torácica se recubrió con espuma flexible expandible, lo que permitió simular el músculo y tejido adiposo que lo rodea.

La presión ejercida sobre los sensores táctiles de presión puede ser variable y su umbral de disparo puede ser calibrado por software o elementos pasivos. Esto permite colocarlos en cualquier punto debajo de la piel sintética, lo que amplía las posibilidades del simulador para registrar sonidos pulmonares, gastrointestinales, entre otros.

Existen diversos simuladores de pacientes disponibles en el mercado; sin embargo, la mayor ventaja competitiva de nuestro desarrollo es la apertura que tiene para realizar modificaciones tanto en su operación (recreación de diferentes situaciones clínicas), como en su estructura y conformación. Además del bajo costo asociado en su fabricación. Esto permite expandir la funcionalidad para simular situaciones clínicas de diferentes órganos o extremidades. Incluso puede expandirse para registrar y monitorear diversas variables fisiológicas relacionadas con la actividad eléctrica muscular, cerebral, respiratoria, pulmonar o mecánica del cuerpo humano.

La futura validación del simulador se realizará en cursos o seminarios donde se practiquen las habilidades de auscultación cardiaca. Esto permitirá evaluar la capacidad del simulador para incrementar los conocimientos teóricos, técnicos, las experiencias o habilidades tanto de médicos como de ingenieros en formación, originando colaboraciones multidisciplinarias que trabajen en disminuir la probabilidad de errores de impericia en la práctica clínica.

\section{AGRADECIMIENTOS}

A DGAPA-UNAM proyecto PAPIME 2019PE109019 "Simulador de Paciente para la Evaluación del Sistema Cardiorrespiratorio" por el financiamiento otorgado para el desarrollo del proyecto. 


\section{REFERENCIAS}

1. Fang JC, O'Gara PT. The history and physical examination: an evidence-based approach. In: Mann DL, Zipes DP, Libby P, Bonow RO, editors. Braunwald's heart disease. 10th ed. Philadelphia: Elsevier Saunders; 2015. pp. 95-113.

2. Sztajzel JM, Picard-Kossovsky M, Lerch R, Vuille C, Sarasin FP. Accuracy of cardiac auscultation in the era of Doppler-echocardiography: a comparison between cardiologists and internists. Int J Cardiol. 2010; 138: 308-310.

3. Kelder JC, Cramer MJ, van Wijngaarden J, van Tooren R, Mosterd A, Moons KG, et al. The diagnostic value of physical examination and additional testing in primary care patients with suspected heart failure. Circulation. 2011; 124: 2865-2873.

4. Mangione S, Nieman LZ. Cardiac auscultatory skills of internal medicine and family practice trainees. A comparison of diagnostic proficiency. JAMA. 1997; 278: 717-722.

5. Mangione S. Cardiac auscultatory skills of physiciansin-training: a comparison of three English-speaking countries. Am J Med. 2001; 110: 210-216.

6. Vukanovic-Criley JM, Criley S, Warde CM, Boker JR, Guevara-Matheus L, Churchill WH, et al. Competency in cardiac examination skills in medical students, trainees, physicians, and faculty: a multicenter study. Arch Intern Med. 2006; 166: 610-616.

7. Gaba DM. The future vision of simulation in health care. Qual Saf Health Care. 2004; 13 (suppl 1): i2-i10.

8. Okuda Y, Bryson EO, DeMaria S Jr, Jacobson L, Quinones J, Shen B, et al. The utility of simulation in medical education: what is the evidence? Mt Sinai J Med. 2009; 76 (4): 330-343.

9. Berkenstadt H, Ziv A, Gafni N, Sidi A. The validation process of incorporating simulation-based accreditation into the anesthesiology Israeli national board exams. Isr Med Assoc J. 2006; 8 (10): 728-733.
10. Reis S, Guimarães P, Coelho F, Nogueira E, Coelho L. 2018 Global Medical Engineering Physics Exchanges/ Pan American Health Care Exchanges (GMEPE/PAHCE) Porto, Portugal: IEEE; 2018. A framework for simulation systems and technologies for medical training; pp. 1-4.

11. Al-Elq AH. Simulation-based medical teaching and learning. J Family Community Med. 2010; 17 (1): 35-40.

12. Karnath B, Thornton W. Auscultation of the heart. Hospital Physician. 2002; 38 (9): 39-45.

13. Gordon MS. Cardiology patient simulator. Development of an animated manikin to teach cardiovascular disease. Am J Cardiol. 1974; 34 (3): 350-355.

14. Champagne MT, Harrell JS, Friedman BJ. Use of a heart sound simulator in teaching cardiac auscultation. Focus Crit Care. 1989; 16 (6): 448-456.

15. Takashina T, Masuzawa T, Fukui Y. A new cardiac auscultation simulator. Clin Cardiol. 1990; 13 (12): 869-872.

16. Akins R, Ho H. Outcomes of using heart sound simulator in teaching cardiac auscultation. Can Med Educ J. 2010; 1 (1): e46-e50.

17. Butter J, McGaghie WC, Cohen ER, Kaye M, Wayne DB. Simulation-based mastery learning improves cardiac auscultation skills in medical students. J Gen Intern Med. 2010; 25 (8): 780-785.

18. McKinney J, Cook DA, Wood D, Hatala R. Simulationbased training for cardiac auscultation skills: systematic review and meta-analysis. J Gen Intern Med. 2013; 28 (2): 283-291.

19. Medical Expo. Simuladores de pacientes de auscultación. Consultado en: https://www. medicalexpo.es/fabricante-medical/simuladorpaciente-auscultacion-15050.html

Correspondencia:

Luis Jiménez-Ángeles

E-mail: luis.jimenez@comunidad.unam.mx 